\title{
Arbor
}

\section{Los hospitales enredados}

\author{
Carlos J. Jiménez Pérez
}

Arbor CLXX, 670 (Octubre 2001), 383-394 pp.

Las iniciativas para reducir costes y aumentar la eficiencia clínica y administrativa, con los sistemas de información en una mano y con los cambios organizativos en la otra, se han sucedido con diverso resultado. $\mathrm{Ni}$ el éxito ni el fracaso han sido nunca totales.

Los centros van a adoptar un modelo colaborativo y convertirse en nudos donde se enlacen las redes básicas de la sociedad de la información: mercado, conocimiento, técnicas y comunicación.

\section{Introducción}

Nada mejor para afrontar el futuro que mirar el pasado, pero quizás sea conveniente para hablar de lo ocurrido leer algo de ciencia-ficción. Así el Hospital Virtual de la Clínica Mayo tiene como visión la siguiente: «En el futuro, los pacientes tendrán la capacidad de registrar y transmitir sus signos vitales al hospital desde sus hogares».

Esto nos ofrece una pista que permite rastrear los pasos que estamos dando con la sinuosa incorporación de la informática a la sanidad. Posiblemente, el aspecto más relevante que está cambiando en la medicina es que el paciente puede dejar de ser el único vehículo válido de la información clínica, ya que en ocasiones preferirá evitar desplazamientos y cuando lo haga irá acompañado de un conocimiento mucho más contrastado sobre su estado salud y forma de mejorarlo para obtener mejores respuestas. La repetición sistemática de pruebas 
diagnósticas, las esperas no justificadas, los continuos saltos entre niveles asistenciales, la atención no personalizada y los errores por falta de organización son aspectos que no querrá comprender y por tanto exigirá su corrección.

Bajo esta perspectiva, es el movimiento de la información necesaria para la realización de la actividad asistencial el que puede servirnos de guía para destacar los «conceptos», huellas y elementos aportados por los sucesivos intentos de informatización de los centros sanitarios.

\section{Conceptos de información, conectividad y comunicación}

En las organizaciones de cualquier sector económico y social se ha considerado la información como un recurso. Sin embargo, considerado en este sentido posee unas características específicas, no comunes con el resto de recursos físicos: crece con el uso (expansión), tiende a transmitirse (difusión) y no se gasta cuando se reparte (inversión).

Por tanto, aparecen unas situaciones excepcionales al tratar de gestionar «el recuso información»(5): necesita almacenarse y limpiarse, adquiere su valor principal cuando se transmite, requiere medidas de seguridad especiales y precisa de agentes que la interpreten y no sólo que la coleccionen.

La calidad de esta gestión junto con los métodos y herramientas para llevarla a cabo han marcado en gran medida los resultados y las diferencias entre los sectores (financiero, industrial, servicios, etc.). Esto ha dado lugar a que actualmente nos encontremos con diversas redes de información que tienen su propio desarrollo y tecnología (p.e. los medios de pago).

A mediados del siglo pasado la ciencia gestó dos concreciones relevantes. Por una parte el recientemente fallecido, matemático e ingeniero, Claude Elwood Shannon publicó en 1948 su Teoría Matemática de la Comunicación cuya terminología en forma de Teoría de la Información se utiliza en la actualidad. Aplicando su famoso Teorema se demuestra que además del mero volumen de datos podemos medir la cantidad de información de un canal (por ejemplo que una imagen de televisión equivale a cien palabras de un buen locutor de radio, no a mil). Por otra parte la URSS en 1957 lanzó el Sputnik, primer satélite terrestre artificial, que nos permitió observar, por primera vez, el mundo globalmente. Estos hechos, junto a otros, permitieron que a finales del siglo XX apareciera un elemento tecnológico que se nos brinda común para diversas actividades humanas: internet. 
Para el entorno sanitario, podemos considerar a internet como una fuente para el conocimiento de los profesionales y, aún más importante, como un vehículo complementario (y en algunos casos alternativo) a la información de salud que cada ciudadano lleva consigo, en su propio cuerpo.

Esta disponibilidad de conexión se traduce en que las organizaciones y profesionales de todos los sectores ya poseen la misma tecnología para gestionar la información y por tanto pueden superar su consideración como recurso para abordarla en el sentido intrínseco: «conocimiento para el pensamiento y la acción». Y el ejemplo más claro se ha producido, precisamente, en la ciencia biomédica con la codificación del Genoma Humano, que nos va a permitir estudiar la vida desde un nuevo punto de vista, el de la información. Una de las primeras consecuencias puede ser que los medios diagnósticos se individualizarán y distribuirán, al menos en su aspecto de toma de datos, para procesarse e interpretarse centralizadamente.

Con internet la capacidad de emitir y recibir mensajes se ha incrementado exponencialmente, produciéndose una fragmentación en los contenidos que exige unas habilidades de integración y síntesis extraordinarias. Esta información en transmisión es, pese a las dificultades de gestión, la que verdaderamente resulta útil. La información reservada (en el cajón) carece de un valor fundamental ya que puede no estar disponible para las personas capaces de mejorarla o que necesiten utilizarla.

El $80 \%$ de los mensajes que intercambian espontáneamente las personas son relativos a la salud y al tiempo atmosférico (compruébese el dato en un ascensor). Ello nos puede dar una idea del potencial de desarrollo que ha de tener la información sanitaria en aspectos como la prevención y promoción de la salud, el conocimiento biomédico, la organización de la asistencia, la equidad de acceso y la eficiencia.

De esta forma, un escenario sanitario futuro, calificable de integrado y cooperante, exigirá una distinta relación entre la Autoridad Sanitaria, las Aseguradoras y los Centros Sanitarios y de estos entre sí. Los centros deberán adoptar un modelo colaborativo y convertirse en nudos donde se enlacen, al menos, 4 redes básicas multisectoriales: (2)

1. Red de asistencia compartida.

2. Red de cadena de suministros en la modalidad de respuesta eficiente al consumidor.

3. Red de servicios diagnósticos y terapéuticos comunes.

4. Red de cooperación para la investigación y obtención de evidencias en resultados. 
Estas tendencias convergen en la promoción de la asistencia compartida. Existe consenso en EEUU y Europa en considerar que se debe disponer de infraestructuras de tecnologías de la información para una sanidad distribuída, basada en la comunidad, con un alto grado de cooperación entre las organizaciones. Por tanto, puede ser útil incorporar aspectos como el trabajo colaborativo y el teletrabajo, en el quehacer diario de nuestros profesionales, ya que la disponibilidad de redes penetrantes y omnipresentes, como Internet, junto con la disponibilidad de potencia de cálculo y comunicaciones asequibles en máquinas como los ordenadores portátiles, los PDA y los teléfonos celulares, está posibilitando un nuevo paradigma de informática y comunicaciones «nómadas».(3)

Con todo ello podemos asegurar que la conectividad ya existe, mientras que la comunicación es una actitud y una aptitud, dos características básicas para el desarrollo de cualquier ciencia y, cómo no, de la medicina: transmitir pensamiento (gnosis) y adquirir conocimiento (praxis).

\section{Huellas de la informatización de los centros sanitarios. Breve Historia}

Hasta mediados de los años 80 nos encontramos con Hospitales y Ambulatorios que, en la mayoría de los casos, únicamente disponían de algunos elementos tecnológicos aislados, para el cálculo y emisión de nóminas, facturas y registros contables (sumadoras, procesadores dedicados de fichas, calculadoras programables). En los servicios asistenciales disponían de sistemas de control empotrados en equipos diagnósticos o de monitorización. Existían algunos hospitales donde excepcionalmente bien la colaboración con alguna facultad de informática o la aportación de algún centro de cálculo patrocinado por alguna gran multinacional informática les había permitido disponer de un gran ordenador donde comenzar el desarrollo del registro de pacientes.

El quinquenio siguiente (1985-1989) vino marcado por la instalación generalizada de los llamados sistemas propietarios multiusuario con una aplicación de registro de pacientes y una aplicación de facturación y por la incorporación de los primeros ordenadores personales (basados en Intel). La distribución del mercado se hizo por cuotas para las diferentes marcas fabricantes al estilo del reparto de la electromedicina. (IBM, PHILIPS, SIEMENS, SPERRY, etc). Aparecieron las conexiones con la Autoridad Sanitaria para los registros de presupuestos y control 
de plantillas. Mejoraron las aplicaciones departamentales suministradas por los laboratorios debido a las necesidades de control de la producción. Algunos servicios clínicos comenzaron a disponer de PCs con programas de gestión de sus pacientes. En 1987 un Hospital incorpora un equipo con sistema operativo UNIX y lo orienta al desarrollo de la gestión de personal y la gestión de pacientes, permitiendo la citación en línea desde los centros de salud directamente sobre las agendas del Centro de Especialidades del Hospital. En 1989, UNIX se declara sistema operativo abierto estándar a nivel europeo, lo que permitió centrarse en los aspectos organizativos, ya que la convergencia tecnológica parecía asegurada.

Durante los 5 años siguientes (1990-1994) se implanta el Plan DIAS «Dotación Informática para la Atención Sanitaria» sistemáticamente en la mayoría de los Centros Sanitarios (Hospitales y Centros de Atención Primaria), con tecnología de sistemas abiertos, sobre un sistema gestor de bases de datos relacional, con un diccionario de datos básico común y con las aplicaciones estándar de gestión de pacientes, de personal y económico-financiera. Los centros se dotan de personal informático de forma independiente y comienza el trabajo de los Grupos de Usuarios para mejorar las aplicaciones entregadas y avanzar en la integración con los servicios diagnósticos de los Centros y en la informatización de la información clínica. Importante fue la creación de la Tarjeta Sanitaria Individual con el objetivo de la identificación única de usuarios del Sistema Nacional de Salud, aunque ya desde el inicio la homogeneidad entre los distintos Servicios de Salud transferidos se vió resentida. Otro elemento característico de este periodo fue la extensión de la Red IRIS a las unidades de investigación de los hospitales a través de la red de Universidades. Ello permitió el acceso de un determinado número de profesionales a internet desde momentos muy tempranos, lo que se tradujo en una educación precoz de los hospitales que accedieron al servicio y necesaria dado el rumbo de la informática sanitaria.

Entre 1995 y el 1999 se provocan múltiples iniciativas destacables desde los Servicios de Salud transferidos (conectividad, gestión económica de los centros, registros clínicos, servicios diagnósticos, integración del Área de Salud, tarjeta sanitaria, centros de soporte, gestión y servicio, portales sanitarios, telemedicina, etc). Adicionalmente se lanzan dos iniciativas de gran calado para el Sistema Nacional de Salud. Por una parte desde el INSALUD se pone en marcha el Plan de Renovación Tecnológica, que viene a crear la dotación en infraestructura de comunicaciones, equipamiento y sistemas necesarios para 
cada centro, a homogeneizar y mejorar tecnológicamente las aplicaciones estándar de los hospitales y centros de salud tras los años de desarrollo individualizado del Plan DIAS, a ofrecer soluciones departamentales integradas y en definitiva a posicionar a los centros en una situación favorable para abordar nuevos retos tras los procesos transferenciales. Por otro lado desde la Secretaría del Estado de Telecomunicaciones y para la Sociedad de la Información del actual Ministerio de Ciencia y Tecnología se lanza el Proyecto PISTA (Promoción e Identificación de Servicios emergentes de Telecomunicaciones Avanzadas) que en su sección de Sanidad ha ofrecido la capacidad a las organizaciones sanitarias de dotarse de las aplicaciones necesarias que la industria no está ofreciendo. Esta propuesta ha sido recogida por distintos centros sanitarios y Servicios de Salud y ha producido diversas aplicaciones en tecnología $i^{*}$ net, multilíngües, basadas en normativa internacional, de aplicación directa en un entorno colaborativo sanitario y de libre utilización por las organizaciones públicas.

También es preciso destacar la labor desarrollada durante estos años tanto por el Comité Europeo de Normalización CEN/TC251 (véase la Tabla 1), y por la Sociedad Española de Informática de la Salud (SEIS), que ha dado la capacidad de interactuar a los profesionales de la salud, a las autoridades sanitarias y a la industria.

Tabla 1

Informática Sanitaria. Extracto de la Normativa actual

\begin{tabular}{|l|l|}
\hline Código de Norma & \multicolumn{1}{|c|}{ Título } \\
\hline ENV 13735: 2000 & $\begin{array}{l}\text { Informática sanitaria. Interoperatividad de los productos sanitarios conectados } \\
\text { a pacientes. }\end{array}$ \\
\hline ENV 13734: 2000 & Informática sanitaria. Representación de la información de los signos vitales. \\
\hline ENV 13728: 2000 & $\begin{array}{l}\text { Informática sanitaria. Interfaces de analizadores clínicos con los sistemas de } \\
\text { información de laboratorios. }\end{array}$ \\
\hline ENV 13607: 2000 & $\begin{array}{l}\text { Informática sanitaria. Mensajes para el intercambio de información en las } \\
\text { prescripciones médicas. }\end{array}$ \\
\hline ENV 12435: 2000 & $\begin{array}{l}\text { Informática sanitaria. Expresión de los resultados de las mediciones en las ciencias } \\
\text { de la salud }\end{array}$ \\
\hline ENV 13729: 2000 & $\begin{array}{l}\text { Informática sanitaria. Identificación segura de usuario. Autenticación fuerte } \\
\text { utilizando tarjetas con microprocesador. }\end{array}$ \\
\hline ENV 12251: 2000 & $\begin{array}{l}\text { Informática sanitaria. Identificación segura de usuario para sanidad. Gestión y } \\
\text { seguridad de la autenticación por contraseñas. }\end{array}$ \\
\hline ENV 13608: 1999 & $\begin{array}{l}\text { Informática sanitaria. Seguridad en las comunicaciones sanitarias. Parte 1: } \\
\text { Conceptos y terminología. Parte 2: Objetos de datos seguros. Parte 3: Canales } \\
\text { de datos seguros. }\end{array}$ \\
\hline
\end{tabular}




\section{Los hospitales enredados}

Tabla 1 (continunación)

Informática Sanitaria. Extracto de la Normativa actual

\begin{tabular}{|c|c|}
\hline Código de Norma & Título \\
\hline ENV 13606: 1999 & $\begin{array}{l}\text { Informática sanitaria. Comunicación de registros clínicos electrónicos. Parte 1: } \\
\text { Arquitectura extendida. Parte 2: Relación de términos del dominio. Parte 3: Reglas } \\
\text { de distribución. Parte 4: Mensajes para el intercambio de información }\end{array}$ \\
\hline ENV 12967: 1998 & $\begin{array}{l}\text { Informática sanitaria. Arquitectura del sistema de información sanitario (HISA). } \\
\text { Parte 1: Capa de middleware sanitaria }\end{array}$ \\
\hline ENV 12924: 1998 & $\begin{array}{l}\text { Informática médica. Clasificación de seguridad y protección para los sistemas } \\
\text { de información sanitarios }\end{array}$ \\
\hline ENV 12612: 1998 & Informática médica. Mensajes para el intercambio de información administrativa sanitaria. \\
\hline ENV 12610: 1998 & Informática médica. Identificación de medicamentos. \\
\hline ENV 12539: 1998 & $\begin{array}{l}\text { Informática médica. Mensajes de solicitud e informes para los departamentos } \\
\text { de pruebas diagnosticas. }\end{array}$ \\
\hline ENV 12264: 1998 & $\begin{array}{l}\text { Informática médica. Estructura de categorías de los sistemas de conceptos. Modelo } \\
\text { de representación semántica. }\end{array}$ \\
\hline ENV 12052: 1998 & Informática médica. Comunicaciones de imágenes sanitarias (MEDICOM). \\
\hline ENV 12018: 1998 & $\begin{array}{l}\text { Informática médica. Estructura de datos de identificación, administrativos y } \\
\text { clínicos, comunes para los dispositivos de conexión intermitente utilizados en } \\
\text { sanidad (incluyendo tarjetas de lectura por máquina). }\end{array}$ \\
\hline UNE 13980: 1988 & $\begin{array}{l}\text { Informática para la salud. Aplicaciones informáticas para personas con } \\
\text { discapacidad. Requisitos de accesibilidad de las plataformas informáticas. Parte } \\
\text { 1: Soporte físico. Parte 2: Soporte lógico. }\end{array}$ \\
\hline ENV 12017: 1998 & Informática médica. Vocabulario de informática medica (MIVoc). \\
\hline ENV 12388: 1997 & Informática médica. Algoritmo para servicios de firma digital en sanidad. \\
\hline ENV 12381: 1997 & Informática médica. Representación del tiempo para problemas específicos en sanidad. \\
\hline ENV 1828: 1996 & $\begin{array}{l}\text { Informática médica. Estructura para la clasificación y codificación de } \\
\text { procedimientos quirúrgicos. }\end{array}$ \\
\hline ENV 1068: 1996 & $\begin{array}{l}\text { Informática médica. Intercambio de información sanitaria. Registro de los sistemas } \\
\text { de codificación }\end{array}$ \\
\hline ENV 1064: & $\begin{array}{l}\text { Informática médica. Protocolo de comunicación normalizado. Electrocardiografía } \\
\text { asistida por ordenador. }\end{array}$ \\
\hline
\end{tabular}

Fuente: AENOR.

\section{Elementos para los próximos años}

\section{A) El nuevo papel de los responsables de tecnologías de la información en los centros sanitarios:}

El sentido de los servicios de informática de los centros está cambiando de forma radical. La externalización de las labores más rutinarias, el hecho de que las aplicaciones troncales (gestión de pacientes, 
de proveedores y de personal) se están centralizando en los servicios de organización de la provisión y en tercer lugar el incremento de la cultura informática de todos los profesionales que creará una tendencia a la internalización o autoinformatización de sus procesos clave, son los tres aspectos que obligan al responsable de los servicios de tecnologías de la información y de las comunicaciones a adoptar un nuevo papel.

Así la función informática deberá organizarse y gestionarse para dar soporte a los retos no sólo tecnológicos sino sectoriales. Deberá demostrar el valor añadido palpable que las TIC pueden proporcionar en el ámbito sanitario, enfatizando en los análisis coste-oportunidad de las inversiones y en el carácter innovador que pueden tomar las soluciones asistenciales basadas en las tecnologías.

Quizás un papel poco desarrollado hasta el momento en los hospitales, en cuanto a tecnologías de la información, es el de la investigación y la docencia. Con herramientas, pero sobre todo con actitudes, de trabajo colaborativo, éste va a ser un campo de actuación trascendental, que el responsable de sistemas de información deberá emprender para que las personas de la organización logren que el conocimiento clave sea aplicado y transmitido.

Otro aspecto que ha de asumir será la responsabilidad de la seguridad de datos, cuestión trascendente en el ámbito sanitario y que va a exigir una cualidad poco común al responsable de tecnologías de la información: la capacidad de hacer compatible la disponibilidad y la seguridad de información, incrementando ambas.

La incorporación de la sanidad a la sociedad de la información, comenzando por la conectividad e intercomunicación entre los profesionales de los centros asistenciales, tendrá que animar buena parte de los esfuerzos de estos responsables, pues el paciente -ciudadano se está convirtiendo en un agente mucho más activo del sistema de información.

Por tanto, empezaremos a encontrar en nuestro centros asistenciales, personas del ámbito de las tecnologías de la información que actuarán como solucionadores de problemas de negocio (6), que saldrán de las salas de ordenadores para apoyar las actividades asistenciales, que tras impulsar la informatización de ciertos procesos comenzarán a gestionarlos y que, en definitiva, van a ejercer funciones cada vez con mayor contenido estratégico.

\section{B) El mercado de las Tecnologías de la Información y las Comunicaciones en Sanidad:}

En Europa, año 2000, el mercado de las Tecnologías de la Información y de las Comunicaciones (TICs) en Sanidad es de 14 billones de euros 
anuales, representando el $6 \%$ del mercado total de las TICs (232 billones de euros / año) y el $2 \%$ de mercado total sanitario (724 billones de euros / año). (4)

Según los expertos este mercado se duplicará en los próximos 5 años si se dan las siguientes condiciones:

- consolidación de la industria mediante fusiones, adquisiciones y colaboraciones

- integración técnica para la oferta de soluciones abiertas, coste-efectivas y con el soporte adecuado

- incremento de la investigación aplicada en TICs dentro de los centros sanitarios

- definición de planes de negocio que aclaren la posición y prioridades de los suministradores

- planificación y presupuestación de las nuevas formas asistenciales: asistencia domiciliaria y teleasistencia

- desarrollo de las redes sanitarias de ámbito regional, mediante el intercambio de mensajes entre los agentes

\section{C) Las Aplicaciones de la Telemedicina:}

El futuro de los sistemas de información sanitaria pasa por satisfacer las necesidades urgentes de interoperabilidad que hagan posible la trasferencia de información entre sistemas informáticos diferentes y dispersos. El establecimiento de estándares en temas tales como registros médicos, formatos, mensajes, códigos, imágenes y documentos multimedia resulta esencial para la adopción y difusión extendida de las aplicaciones telemáticas para la salud.

Las siguientes aplicaciones se incluyen dentro del campo de aplicación actual de la telemedicina, si bien existen factores inhibidores como la ausencia de un sistema de facturación, el marco legal exigible y el rechazo a realizar un juicio clínico sin la presencia física del paciente. (4).

- Tele-educación: Una de las principales aplicaciones en el entorno hospitalario será la formación de postgrado para profesionales sanitarios, especialmente para los médicos. Para facilitarla se precisa un ajuste de la programación, y una mejora y expansión de la tecnología móvil.

- Tele-contactos: Las reuniones entre dos o más agentes sanitarios que hasta ahora se han realizado con un éxito relativo a través del teléfono y teleconferencias ad-hoc, sin embargo pueden generalizarse a través de las redes comunes y herramientas simples 
como correo electrónico, chat y tele-conferencia. En el caso de la tele-consulta, es particularmente útil para la búsqueda de segunda opinión entre colegas para patologías complejas.

- Tele-emergencia: La interconexión de los diferentes servicios de alerta y emergencia, así como el acceso a expertos remotos para situaciones críticas deberán soportar mecanismos para la toma de decisiones, como por ejemplo triage, planificación de traslados, etc.

- Tele-diagnóstico: Se refiere a aquellas aplicaciones en que la prueba diagnóstica se realiza con la participación de un experto ubicado físicamente a distancia. Ejemplo de ello son tele-electrocardiogramas o tele-endoscopias. Serán de especial desarrollo para aquellas pruebas en que la muestra pueda viajar electrónicamente y se precise un equipamiento específico con gran capacidad de cálculo como, por ejemplo, en los estudios genéticos.

- Tele-cirugía: Si bien las intervenciones quirúrgicas a distancia han tenido una cierta popularidad debido a algunas experiencias en situaciones especiales y muy controladas, la aplicación más importante puede venir de la llamada cirugía asistida por ordenador, en la que se produce una visualización virtual del área del cuerpo a intervenir y las correspondientes tele-consultas a expertos durante la operación.

- Tele-monitorización: Su aplicación puede producirse en aquellos programas a gran escala en que el diagnóstico experto y el equipamiento tecnológico se encuentra concentrado en unos pocos centros de referencia, como por ejemplo la monitorización de pacientes diabéticos para la detección precoz de glaucoma y el seguimiento de pacientes en hospitalización domiciliaria.

\section{D) La redes sanitarias regionales}

La sanidad moderna no se provee por una institución o por un grupo de profesionales de forma aislada. La sanidad actual se ha de suministrar mediante la estrecha cooperación entre múltiples centros sanitarios y grupos de profesionales, trabajando juntos y utilizando su conocimiento experto en el esfuerzo común de proporcionar el servicio de mejor calidad y coste-efectividad posibles. Sin ello resultaría difícil aplicar las técnicas avanzadas de las diferentes especializaciones que actualmente proveen los hospitales, los centros de salud, los centros diagnósticos, la asistencia domiciliaria, los servicios de emergencia y los servicios sociales.(1) 
Aunque en Europa aún no existen como tales, las redes sanitarias regionales deberían proporcionar al menos los siguientes servicios:

- Comunicación diaria de prescripciones, resultados diagnósticos biomédicos, etc.

- Sistemas de correo electrónico seguro sobre la información relacionada con los pacientes

- Registros comunes de admisión para hospitales y centros diagnósticos

- Registros médicos compartidos

- Sistemas de alerta y emergencia

- Facilidades de telemedicina

- Protocolos y vías clínicas para tratamientos completos y la continuidad asistencial

- Sitios web de información sanitaria para profesionales, pacientes y ciudadanos

- Información administrativa del conjunto y sistemas de gestión global

Afortunadamente, la penetración de Intenet en la sociedad va a permitir que los proyectos de desarrollo de estas redes regionales puedan llevarse a la práctica. En concreto, para facilitar este proceso de iniciación se han de considerar las tecnologías de la seguridad y de la interoperatividad de forma que, combinadas con los protocolos de comunicaciones de Internet, puedan cubrir necesidades mediante el intercambio de mensajes en el entorno sanitario (véase Tabla 2).

Tabla 2

Servicios y Tipos de Mensajes en la Red Regional Sanitaria

\begin{tabular}{|l|l|l|}
\hline Servicios Clínicos & Servicios Sanitarios & Servicios Administrativos \\
\hline Mensajes clínicos & Información de vigilancia & Cobros \\
e-mail clínico & Páginas amarillas & Comercio electrónico \\
Admisión asistencial & Vías clínicas profesionales & Identificación de Pacientes \\
Registros compartidos & Gestión clínica & Gestión de recursos \\
Protocolos de cuidados & Información sanitaria pública & \\
$\begin{array}{l}\text { Movilidad y emergencia } \\
\text { Monitor hospitalización a domicilio } \\
\text { Telemedicina }\end{array}$ & Desarrollo profesional continuado & \\
\hline
\end{tabular}

Fuente: John Oates y otros.

Para facilitar este proceso, se hace imprescindible una estrategia común en los niveles regionales, nacionales y europeo. 


\section{Conclusión}

En definitiva, en los hospitales enredados, la informática emergente va a ayudar a gestionar mejor las interrelaciones, el desarrollo de redes virtuales modificará la provisión de servicios, los servicios integrarán la telemática en el desarrollo de sus procesos y, finalmente, nuevos profesionales y nuevos gestores deberán adquirir habilidades relacionadas con la utilización de las nuevas tecnologías.

\section{Bibliografía}

1 OAtes, J., BJERREGAARD, H. Building Regional Health Care Networks in Europe. IOS Press. Netherlands. 2000.

2 MARIMón, S. La sanidad en la sociedad de la información. Díaz de Santos. cop. Madrid 1999.

3 BARRY, M. y otros. A Brief History of Internet, On The Internet. Internet Society. Mayo/Junio 1997.

4 Health Information Society Technology Based Industry Study. Deloitte \& Touche. 2000.

5 Rodenes, M. Sistemas de información para la gestión. Univ. Politécnica de Valencia. 1989.

6 JiméneZ, C. y otros. El papel de los Servicios de Informática en los Hospitales del año 2002. Informes SEIS. Madrid. 1999. 